\title{
TECNOLOGIAS DA INFORMAÇÃO E COMUNICAÇÃO NO ENSINO E APRENDIZAGEM DA ANATOMIA HUMANA: UM RELATO ACADÊMICO EM TEMPOS DE COVID-19
}

\author{
INFORMATION AND COMUNICATION TECHNOLOGIES IN TEACHING AND LEARNING HUMAN \\ ANATOMY: AN ACADEMIC REPORT IN TIMES OF COVID-19
}

\section{Janaína Damascena da Silva Souzaa, Rafael Virgens dos Santosa, Maria Oliveira Penha Belémb ${ }^{b}$ Leila Valverde Ramosa}

\author{
Centro Universitário de Salvador- UNICEUSAa \\ Universidade Federal da Bahia - UFBA \\ E-mail: janaina.janadss@gmail.com
}

\section{RESUMO}

Este relato de experiência surgiu a partir dos desafios enfrentados pela comunidade acadêmica diante da migração do estudo da Anatomia Humana presencial para as aulas online de caráter emergencial em tempos de COVID-19. As tecnologias de informação e comunicação (TIC'S) foram incorporadas ao ensino na tentativa de dar continuidade às aulas presenciais nas Instituições de Ensino Superior (IES). A experiência ocorreu no Centro Universitário de Salvador (UNICEUSA), vinculado ao projeto de extensão do Departamento de Biomorfologia da Universidade Federal da Bahia (UFBA) intitulado "Metodologias ativas no ensino da Anatomia Humana", no período de Março a Julho de 2020. A pandemia da COVID-19 trouxe mudanças e debates significativos para o ensino da Anatomia Humana, já que ensinar e aprender tornou-se um processo mais dinâmico e interativo, mudando de forma irreversível a autonomia dos discentes e a práxis docente.

Palavras-chave: Acesso às TIC; educação a distância; infecções por Coronavírus.

\section{ABSTRACT}

This experience report arose from the challenges faced by the academic community in the face-to-face human anatomy study migration to emergency online classes in times of COVID-19. Information and communication technologies (ICT's) have been incorporated into teaching in an attempt to continue face-to-face classes in higher education institutions (HEIs). The experiment took place at the University Center of Salvador (UNICEUSA), linked to the extension project of the Department of Biomorphology of the Federal University of Bahia (UFBA) entitled "Active methodologies in the teaching of Human Anatomy", from March to July 2020. The COVID-19 pandemic brought significant changes and debates to the teaching of Human Anatomy, since teaching and learning became a more dynamic and interactive process, irreversibly changing the automaton of students and teaching praxis.

Keyword: ICT access; distance education; Coronavirus infections 


\section{INTRODUÇÃO}

O isolamento social causado pela pandemia por conta do novo coronavírus (SARS-CoV-2), gerou algumas modificações, principalmente na esfera educacional. O início do ano de 2020 foi marcado pelo surto de uma pneumonia até então desconhecida, sendo seu primeiro caso registrado em dezembro de 2019 na cidade de Wuhan, província de Hubei, na China (WHO, 2020).

Nesse sentido, a Organização Mundial de Saúde (OMS) declarou em 30 de janeiro de 2020, que o referido surto foi causado pelo novo coronavírus (SARS-CoV-2) e a doença passou a ser denominada COVID-19. A partir daí, constituiu-se como uma emergência de saúde pública de importância internacional, o mais alto nível de alerta da organização, conforme previsto no Regulamento Sanitário Internacional (UNASUS, 2020).

Segundo a Organização Pan-Americana da Saúde (OPAS, 2020), em 11 de março de 2020, a COVID-19 foi caracterizada pela OMS como uma pandemia. Mundialmente, foram confirmados 63.360.234 casos de COVID-10 e 1.475 .825 mortes até 2 de dezembro de 2020 (OPAS, 2020).

Os impactos da pandemia do novo coronavírus tiveram como plano de ação para a maioria dos países, a adoção de estratégias temporárias de isolamento social, repercutindo assim em um quadro majoritário de fechamento presencial das unidades escolares ao longo do tempo, atingindo 317.816.657 estudantes no período de 03 de dezembro de 2020 (UNESCO, 2020).

No dia 18 de março, o Diário Oficial da União (DOU) publicou a portaria $\mathrm{N}^{\circ} 343$ que dispõe sobre a substituição das aulas presenciais por aulas em meios digitais enquanto durasse a situação de pandemia da COVID-19 (BRASIL, 2020). Com isso, houve uma necessidade de ressignificar o ensino presencial dando espaços para as Tecnologias da Informação e Comunicação (TIC's), realçando a sua importância no contexto educacional, alavancando reflexões sobre o ensino tradicional e o atual ensino online de emergência.

$\mathrm{O}$ objetivo desse trabalho foi relatar a experiência vivenciada através do ensino online nas aulas de Anatomia Humana numa instituição de ensino superior (IES) em tempos de pandemia da COVID-19 durante o período de março a julho de 2020.

Antes da pandemia, a disciplina de Anatomia Humana tinha um caráter teórico-prático, o que motivava e envolvia bastante os acadêmicos. Era ofertada presencialmente, de forma teórica em sala de aula, e prática, no laboratório de Anatomia Humana da instituição para os cursos de Fisioterapia, Enfermagem, Nutrição e Estética. A carga horária era composta de 60 horas totais, sendo distribuída em 30 horas teóricas e 30 horas práticas.

Como recursos pedagógicos nas aulas teóricas, utilizava-se o programa Microsoft Power Point para explanação do conteúdo e livros de Anatomia Humana disponíveis na biblioteca física e virtual da faculdade. Posteriormente, os alunos eram encaminhados ao laboratório para visualização das estruturas apresentadas nas maquetes anatômicas.

Esse relato justifica-se pela relevância do tema abordado, compartilhando a experiência da migração do ensino tradicional para o ensino online. Houve a necessidade de implementação das TIC's, tornando o discente membro ativo do processo de ensino e aprendizagem, bem como a necessidade de se rever antigos conceitos sobre educação e da práxis docente. A discussão acerca do tema pode auxiliar a solucionar problemas aqui destacados e mostrar dificuldades que posteriormente poderão se transformar em aprendizado.

\section{METODOLOGIA}

O presente trabalho trata-se de um relato de experiência, no qual foi utilizada a metodologia descritiva. Para Fernandes e Gomes (2003), a metodologia descritiva pode ser utilizada para avaliar programas e podem ou não formular hipóteses, servindo também de base para estudos de relações causais. Para fundamentação teórica e confrontamento dos dados da literatura com a experiência vivenciada no decorrer do semestre, foi realizada uma busca de artigos científicos na base de dados Pubmed, SciElo e Google Acadêmico.

Desenvolveu-se uma proposta didática numa instituição de ensino superior (IES) localizada em Salvador-BA, o Centro Universitário de Salvador (UNICEUSA), no período de março a julho 2020. O presente trabalho esteve vinculado ao projeto de extensão do Departamento de Biomorfologia da Universidade Federal da Bahia (UFBA) intitulado "Metodologias ativas no ensino da Anatomia Humana".

Para consecução das atividades, utilizou-se a plataforma ZOOM meeting institucional (com tempo ilimitado de utilização) para os encontros síncronos, cujos dados de acesso eram disponibilizados no portal da Universidade em datas pré-agendadas. Esses encontros 
foram realizados no formato online ao vivo com a docente da disciplina Anatomia Humana durante 90 minutos na plataforma.

Acerca dos recursos didáticos utilizados nas aulas online, para além do tradicional Microsoft Power Point, a professora utilizou TIC's tais como imagens retiradas de atlas virtuais de anatomia humana devidamente licenciados e disponibilizou casos clínicos, que foram discutidos e corrigidos durante os encontros síncronos.

No que concerne às atividades assíncronas, os acadêmicos recebiam e-mails com sugestões de atividades complementares a serem realizadas nas aulas de Anatomia Humana, como resolução de exercícios, links de vídeos e atividades de fixação dos conteúdos abordados.

\section{RESULTADOS}

Visando alcançar os objetivos do estudo, elucidar de que forma as TIC's podem ser utilizadas como técnicas pedagógicas facilitadoras no processo de ensino e aprendizagem nas aulas de anatomia humana, buscou-se realizar diversas atividades ao longo do semestre online emergencial. Gusso et al (2020) ressaltam que a pandemia do novo Coronavírus impôs grandes mudanças na forma de viabilizar o acesso ao ensino superior. Acerca dessa temática, Pasini et al (2020) acrescentam que a migração do ensino tradicional para o ensino online ocasionou mudanças abruptas em um semestre atípico, o qual trouxe à tona a necessidade de reflexões sobre o modelo de ensino vigente no Brasil.

As aulas online do presente estudo ocorreram numa turma constituída por 20 acadêmicos regularmente matriculados no curso de Fisioterapia do Centro Universitário de Salvador (UNICEUSA) na cidade de Salvador-BA, sendo 5 do sexo masculino e 15 do sexo feminino. Os encontros síncronos aconteceram com assistência do professor, sanando as dúvidas no momento em que surgiam, por vídeo ou por chat corroborando com Pretto et al (2020), o qual afirma que o ensino deve ser colaborativo e interativo, fortalecendo o potencial crítico, criativo, pautado em valores democráticos, estimulando o desenvolvimento de propostas educativas que possibilitem a incorporação das TIC's nas práticas pedagógicas em todos os níveis educacionais numa perspectiva não instrumental.

Relativamente às fragilidades no ensino online, o quadro 1 apresenta as principais vivenciadas no presente estudo bem como as suas possíveis soluções.

Quadro1. Fragilidades no ensino online e possíveis estratégias de soluções

Fragilidades do Ensino online

Dificuldade de comunicação entre discentes e docentes pela ausência de contato presencial

Carência de infraestrutura física

Carência de recursos tecnológicos

Instabilidade da internet

Ausência do laboratório de Anatomia Humana

\section{Estratégias de resolução}

Utilização de ambientes virtuais de aprendizagem como o Google sala de aula e Microsoft Teams

Ajustar a ergonomia do local de estudo

Buscar apoio na universidade ou do estado

Ficar o mais próximo possível do local que o sinal da internet for mais potente

Utilização de Atlas virtual de Anatomia Humana; 
Acerca da temática ergonomia, observou-se que nas IES privadas houve uma transferência dos cursos para as redes mesmo com a dificuldade em manter um espaço isolado adequado para o estudo com mobiliário ergonomicamente desenhado. Além disso, constatou-se a falta de equipamentos para que fosse possível realizar com comodidade e tranquilidade, as aulas online. De acordo com Cavalcante et al (2020), a falta ou acesso precário à internet fragilizam o processo de ensino e aprendizagem, nem todos os discentes e docentes estavam preparados para a inserção das novas tecnologias.

A ausência do laboratório de anatomia humana também se caracterizou como uma dificuldade no ensino e aprendizagem em tempos de pandemia. De acordo com Carneiro (2020), apenas o uso das TIC's não é suficiente, nem garantia de uma boa aprendizagem. Cavalcante et al (2020) defendem o uso das TIC's como estratégia de caráter complementar e não substitutivo ao ensino presencial, pois pode afetar algumas competências necessárias aos graduandos da área da saúde.

Observou-se que as TIC's possuem um grande potencial de complementação do estudo da Anatomia Humana, no entanto, ficar muito tempo em frente do computador ou celular ocasiona cansaço e desconforto físico, fazendo com que a aula não tenha um bom rendimento. De acordo com Reis et al (2020), um dos fatores limitantes do ensino online é a dificuldade dos acadêmicos manterem a atenção durante a aula, bem como não poderem discutir com os colegas de classe, nem tirarem dúvidas com o professor de forma presencial, como é tradicionalmente preconizado.

Soma-se a isso, a questão biopsicossocial relacionada à pandemia da COVID-19, atrelada à preocupação em não ser contaminado pelo SARS-CoV2, redução da renda familiar, aumento das taxas de desemprego e alta nos preços dos produtos, custo de vida de uma forma geral. Gusso et. al. (2020) corroboram com essa questão ao realçarem dificuldades como enfrentamento dos casos de adoecimento por COVID-19 no domicílio e na família dos discentes, associado ao estresse gerado em decorrência do distanciamento social.

Acerca dos recursos utilizados nas aulas online, observou-se a disponibilização via e-mail de vídeos curtos apresentando as estruturas das maquetes anatômicas de acordo com o roteiro das aulas práticas da disciplina presencial. Com autorização da IES e respeitando todos os protocolos sanitários previstos por conta do isolamento social da COVID-19, os vídeos foram gravados pela docente no laboratório de Anatomia da faculdade de forma a tentar maior interatividade com os alunos e uma aproximação com as aulas práticas, tão prejudicadas nesse período de pandemia. O recurso didático teve boa receptividade por parte dos alunos, sendo pedagogicamente útil, melhorando a aprendizagem. Bastos et al (2020) apontam em seu relato que a estratégia da disponibilização de vídeos facilitou a compreensão do conteúdo dos alunos.

No que refere às avaliações, ocorreram de forma processual e a pontuação distribuída em duas etapas: etapa 1 e etapa 2 . Na etapa 1 foram realizadas atividades participativas com peso 4 , envolvendo uma avaliação, disponibilizada via e-mail contendo questões de múltipla escolha e uma questão específica envolvendo metodologias ativas. Nesse sentido os alunos foram orientados à criação de um jogo anatômico educacional que pudesse facilitar a aprendizagem ativa da disciplina de anatomia humana, visando estimular a criatividade, pesquisa e interatividade. A entrega da avaliação foi via e-mail, com prazo de 7 dias. Já na etapa 2 os alunos realizaram uma única avaliação no formulário Google forms com peso 6, composta de 10 questões ( 9 perguntas de múltipla escolha e 1 dissertativa).

A segunda etapa da avaliação foi realizada no final do semestre, incluindo todos os assuntos abordados. O link de acesso foi disponibilizado no chat do aplicativo Zoom na aula síncrona e os alunos tiveram duas horas para sua conclusão. De uma forma geral, não ocorreram dificuldades para a realização da atividade avaliativa pois houve uma explicação prévia de como a mesma seria realizada e a docente estava disponível na sala virtual em caso de eventuais dúvidas. A grande vantagem da avaliação realizada no Google forms é que com apenas alguns cliques a atividade estava disponível para preenchimento, poderia ser finalizada e o seu feedback era dado imediatamente após o preenchimento. Outro aspecto importante é que através das TIC's não há necessidade de impressões dessas avaliações, sendo altamente sustentável do ponto de vista ambiental.

Entretanto, a fragilidade desse tipo de avaliação está relacionada ao acesso à internet, que muitas vezes se encontrava instável ou inexistente. O barulho nos domicílios causados por familiares que também compartilham do isolamento social e da vizinhança são fatores que pode atrapalhar o desempenho do aluno.

Através da realização da prova é possível mensurar os conhecimentos adquiridos pelos alunos no decorrer do semestre. $\mathrm{O}$ resultado da avaliação pode mostrar possíveis falhas nas metodologias de ensino utilizadas pelos docentes e assim, ajustá-las para melhores resultados futuros e também pode mostrar 
falhas na organização dos estudos dos discentes.

No que diz respeito à gravação das aulas, estas não foram gravadas e disponibilizadas para os discentes devido aos direitos autorais dos mesmos, já que no contrato assinado não há nenhuma cláusula que permita o uso de sua imagem. O Ministério Público do Trabalho publicou no dia 17 de julho de 2020 uma norma técnica sobre a defesa da saúde e dos direitos fundamentais de professores quanto ao trabalho em plataformas virtuais no período da pandemia da COVID-19 que pró́be fotografar, gravar, compartilhar e divulgar a imagem ou o material de conteúdo autoral do professor, evitando assim o uso indevido dos seus direitos de imagem e direitos autorais (BRASIL, 2020).

Garcia et al (2020) acrescentam que os discentes adquiriram maior autonomia, maior senso crítico, e que ainda melhoraram a escrita de forma significativa. No presente estudo observou-se que a experiência do ensino online traz também a praticidade para os alunos de não terem que se deslocar de casa para a IES, aumentado assim o tempo disponível para o estudo.

Após experiência vivenciada na disciplina de anatomia humana, verificou-se que o ensino online proporcionou ao aluno maior aproximação das TIC's, transformando-o em membro ativo do seu aprendizado, facilitando a absorção das competências necessárias para exercer as suas atividades estudantis, profissionais e de cidadania. Costa et Al. (2019) corroboram com essa ideia, ao afirmarem que o discente se torna ativo e autônomo no processo de aprendizagem, já que consegue interagir com diferentes pessoas e culturas, proporcionando uma visão de mundo mais crítica $\mathrm{e}$ diferente.

\section{CONCLUSÃO}

As transformações causadas pela pandemia do novo Coronavírus trouxeram mudanças irreversíveis no ensino e aprendizagem da anatomia humana. É um momento de desafios e adaptações para toda a comunidade acadêmica, mas também é uma fase de aprendizado e revisão de antigos conceitos. Os docentes foram obrigados a se reinventarem e utilizarem novas metodologias de ensino e os discentes a adquirirem maior autonomia e utilização de novas ferramentas de aprendizagem.

Ficou evidente que a utilização das TIC's como instrumentos de ensino e aprendizagem da anatomia complementaram o ensino em tempos de COVID-19, pois foi possível dar continuidade ao ano letivo, proporcionando benefícios aos docentes e discentes. No entanto, as TIC's não substituem as atividades práticas realizadas no laboratório.

Espera-se, portanto que o presente trabalho possa contribuir no desenvolvimento de propostas educativas que possibilitem a incorporação das TIC's nas práticas pedagógicas em todos os níveis educacionais, especialmente na educação superior relacionada ao estudo da anatomia humana.

\section{REFERÊNCIAS}

BARBOSA, A. M.; VIEGAS, M. A. S.; BATISTA, R. L.N. F. F. Aulas presenciais em tempos de pandemia: relatos de experiências de professores do nível superior sobre aulas remotas. Revista Augustus, Rio de Janeiro, v. $25, \quad$ n. 51, p. 255-280, jul./out. 2020. DOI: https://doi.org/10.15202/1981896.2020v25n51p25 5.

Disponível em: https://revistas.unisuam.edu.br/index.php/revistaau gustus/article/view/565. Acesso em: Agosto 2020.

BARRETO, A. C. F.; ROCHA, D. S. Covid 19 e educação: resistências, desafios e (im)possibilidades. Revista Encantar - Educação, Cultura e Sociedade, Bom Jesus da Lapa, v. 2, p. 01-11, jan./dez. 2020. DOI: http://dx.doi.org/ 10.46375/encantar.v2.0010.

Disponível em: https://observatoriodeeducacao.institutounibanco.or g.br/cedoc/detalhe/tfr-covid-19-e-educacaoresistencias-desafios-e-im-possibilidades, 27bc544b8d10-4a9e-be20-0b7dd03f36f4. Acesso em: Agosto 2020.

BASTOS, M. C. et. Al. Ensino remoto emergencial na graduação em enfermagem: relato de experiência na covid-19. Revista Mineira de Enfermagem, v. 24, p. e1335, 2020. DOI: 10.5935/1415-2762.20200072. Disponível em: https://cdn.publisher.gn1.link/reme.org.br/pdf/e_1335.p df. Acesso em: Outubro 2020. 
BASTOS, M. C. et. Al. Ensino remoto emergencial na graduação em enfermagem: relato de experiência na covid-19. Revista Mineira de Enfermagem, p. 111, 2020. DOI PROVISÓRIO: 10.5935/14152762.20200072.

Disponível em: https://cdn.publisher.gn1.link/reme.org.br/pdf/e_13 35.pdf. Acesso em: Agosto 2020

BRASIL. Decreto $\mathrm{n}^{\circ} 343$, de 17 de março de 2020. Regulamenta o art. 80 da Lei $\mathrm{n}^{\circ}$ 9.394, de 20 de dezembro de 1996, que estabelece as diretrizes e bases da educação nacional. Disponível em: https://www.in.gov.br/en/web/dou/-/portaria-n-343de-17-de-marco-de-2020-248564376. Acesso em: Agosto 2020.

CARNEIRO, L. A. RODRIGUES, W.; FRANÇA, G.; PRATA, D. N. Uso de tecnologias no ensino superior público brasileiro em tempos de pandemia COVID-19. Research, Society and Development, v. 9, n. 8, p. e267985485, 2020. DOI: https://doi.org/10.33448/rsdv9i8.5485.

Disponível em: https://rsdjournal.org/index.php/rsd/article/view/54 85. Acesso em: N[ovembro 2020.

CAVAlCANTE, A. S. P. et. Al. Educación superior en salud: educación a distancia en medio de la crisis del nuevo coronavirus en Brasil. Avances en Enfermería, Colombia, v. 38, n. 1, p. 113-121, 2020. DOI: https://doi.org/10.15446/av.enferm.v38n1su pl.86229. Disponível em: https://revistas.unal.edu.co/index.php/avenferm/arti cle/view/86229. Acesso em: Agosto 2020.

CORDEIRO, M. V. C.; COELHO, N. B.; SARAIVA, P. M.; RODRUIGUES, T. A.; PINHEIRO, A. A. G. Os Novos Desafios dos Professores de IES no Pós Pandemia: Um Estudo Realizado Com Docentes das Instituições de Ensino Superior de Juazeiro do Norte Ceará. Revista Multidisciplinar e de Psicologia, v. 14, n. $52, \quad$ p. $703-717,2020$. DOI: 10.14295/idonline.v14i52.2749. Disponível em: https://idonline.emnuvens.com.br/id/article/view/27 49/0. Acesso em: Outubro 2020.

Da COSTA, J. D.; SANTOS, W. L.; Da SILVA, J. S.; ALVES, M. M. S. Tecnologias e educação: o uso das TIC como ferramentas essenciais para o processo de ensino e aprendizagem. Brazilian Journal of Development, Curitiba, v. 5, n. 11, p. 25034-25042, 2019. DOI: $\quad 10.34117 /$ bjdv5n11-177. Disponível em: https://www.brazilianjournals.com/index.php/BRJ D/article/view/4627. Acesso em: Agosto 2020.

Da SILVA, M. L. A.; ASSIS, L. M. Diálogos abertos em avaliação educacional: um relato de experiência de um grupo de estudos durante a pandemia da covid-19. Boletim de Conjuntura, Boa Vista, v. 3, n. 8, p. 48-56, 2020. DOI: http://doi.org/10.5281/zenodo.3955236.

Disponível

em: https://revista.ufrr.br/boca/article/view/MeireLucia. Acesso em: Setembro 2020.

De OLIVEIRA, H. V.; De SOUZA, F. S. Do conteúdo programático ao sistema de avaliação: reflexões educacionais em tempos de pandemia (covid-19). Revista Boletim de Conjuntura, Boa Vista, v. 2, n. 5, p. $15-24$ 2020 .

DOI: http://dx.doi.org/10.5281/zenodo.3753654.

Disponível

em: https://revista.ufrr.br/boca/article/view/OliveiraSou za. Acesso em: Outubro 2020

FAVERO, M.; FERREIRA, T. J.; GOIS, A. L. A. Ensino Superior em Saúde em tempos de Pandemia: reflexões emergentes. Brazilian Journal of Development, Curitiba, v. 6, n. 10, p. 79345-79353, 2020. DOI: 10.34117/bjdv6n10-387.

Disponível em: https://www.brazilianjournals.com/index.php/BRJ D/article/view/18443. Acesso em : Outubro 2020.

FEITOSA, A. N. A. et. Al. Tecnologias educacionais em tempos de pandemia: relato de experiência. Brazilian Journalof Production Engineering, São Mateus, v. 6, n. 6, p. 166-172, 2020. Disponível em: https://periodicos.ufes.br/bjpe/article/view/32539.

Acesso em: Novembro 2020.

FERNANDES, L. A.; GOMES, J. M. M. Relatórios de pesquisa nas ciências sociais: características e modalidades de investigação. Revista ConTexto, Porto Alegre, v. 3, n. 4 . Disponível em: https://www.seer.ufrgs.br/ConTexto/article/view/11 638. Acesso em: Agosto 2020.

FERREIRA, G. S. M.; VASCONCELOS, L. C. A.; FERREIRA, F. I. S.; OLIVEIRA, P. M. C.; De PAULA, P. H. A. Pandemia do COVID-19 e as possibilidades de ressignificação das atividades de gestão no ensino superior: relato de experiência. Revista Enfermagem Atual In Derme, v. 93 (2020): ESPECIAL COVID19. DOI: https://doi.org/10.31011/reaid-2020-v.93-n.0- 
$\operatorname{art.814.~}$

Disponível

em: http://www.revistaenfermagematual.com/index.php /revista/article/view/814. Acesso em: Setembro 2020.

GARCIA, L. R; De JESUS, B. A.; MENEZES, L. M. S.; De MENDONÇA, M. H. R. Como a pandemia pelo novo Coronavírus afetou o desenvolvimento dos discentes do $5^{\circ}$ semestre de biomedicina de um centro universitário do Estado do Pará: Um relato de experiência. Brazilian Journal of health Review, Curitiba, v. 3, n. 4, p. 81458154 Jul/Ago 2020. DOI:10.34119/bjhrv3n4-074. Disponível

em: https://www.brazilianjournals.com/index.php/BJH R/article/view/13149. Acesso em: Setembro 2020.

GOMES, V. T. S. et. Al. A Pandemia da Covid-19: Repercussões do Ensino Remoto na Formação Médica. Revista Brasileira de Educação Médica, Brasília, v. 44, n. 4, p. e-114, 2020. DOI: https://doi.org/10.1590/1981-5271v44.4-

20200258. Disponível em: https://www.scielo.br/scielo.php?pid=S0100-

55022020000400602\&script $=$ sci arttext. Acesso em: Setembro 2020.

GUSSO, H. L. et. Al. Ensino superior em tempos de pandemia: diretrizes à gestão universitária. Revista Educação e Saúde, Campinas, v. 41, p. e238957, 2020. DOI: https://doi.org/10.1590/es.238957. Disponível em: https://www.scielo.br/scielo.php?script=sci arttext \&pid=S0101-73302020000100802\&tlng=pt. Acesso em: Novembro 2020.

JOYE, C. R.; MOREIRA, M. M.; ROCHA, S. S. D. Educação a Distância ou Atividade Educacional Remota Emergencial: em busca do elo perdido da educação escolar em tempos de COVID-19. Research, Society and Development, v. 9, n. 7, p. e521974299, 2020. DOI: https://doi.org/10.33448/rsd-v9i7.4299.

Disponível

em: https://rsdjournal.org/index.php/rsd/article/view/42 99. Acesso em: Novembro 2020.

MINISTÉRIO PÚBLICO DO TRABALHO (MPT). Nota técnica - GT COVID-19 - 11/2020. Disponível em: https://mpt.mp.br/pgt/noticias/nota-tecnica-n-112020-trabalho-on-line-de-professores-gt-covid-19mpt.pdf. Acesso em: Agosto 2020

ORGANIZAÇÃO PAN-AMERICANA DA SAÚDE (OPAS). OMS afirma que COVID-19 agora é caracterizada como pandemia. OPAS, 11 de mar. 2020.

Disponível

em: https://www.paho.org/bra/index.php?option=com c ontent\&view=article\&id=6120:oms-afirma-que-covid19-e-agora-caracterizada-como-pandemia\&Itemid=812. Acesso em: Agosto 2020.

PASINI, C. G. D.; De CARVALHO, E.; ALMEIDA, L. H. C. a educação híbrida em tempos de pandemia: algumas considerações. Observatório Socioeconômico da COVID-19, $2020 . \quad$ Disponível em: https://www.ufsm.br/app/uploads/sites/820/2020/06 /Textos-para-Discussao-09-Educacao-Hibrida-emTempos-de-Pandemia.pdf. Acesso em: Outubro 2020

PRETTO, N. L.; BONILLA, M. H. S.; SENA, I. P. F. S. Educação em tempos de pandemia: reflexões sobre as implicações do isolamento físico imposto pela COVID19. Salvador, Edição do autor, 18 p., 2020.

REGUEIRO, E. M. G. et. Al. Ensino mediado por tecnologias no curso de Fisioterapia do Centro Universitário Barão de Mauá durante o período de pandemia da COVID-19. Revista Interdisciplinar de Saúde e Educação, Ribeirão Preto, v. 1, n. 1, p. 108-119, 2020. Disponível

em: https://periodicos.baraodemaua.br/index.php/cse/art icle/view/36. Acesso em: Agosto 2020.

REIS, E. F.; ROCHA, V. M. P.; Da SILVA, C. G. L. Avaliação do ensino remoto de Epidemiologia em uma universidade pública do Sul do Brasil durante pandemia de COVID-19. Scientific Electronic Library Online. DOI: https://doi.org/10.1590/SciELOPreprints.1152. Disponível

em: https://preprints.scielo.org/index.php/scielo/preprint /view/1152. Acesso em: Outubro 2020.

SCHEUNEMANN, C. M. B.; De ALMEIDA, C. M. M.; LOPES, P. T. C. Digital Technologies in the teaching and learning of human anatomy: analysis of the perceptions of higher education academics. Acta Science, $\quad$ v. $\quad 21, \quad$ n. $\quad 1$, 2019. DOI: https://doi.org/10.17648/acta.scientiae.v21is s1id4798.

Disponível em: http://www.periodicos.ulbra.br/index.php/acta/artic le/view/4798. Acesso em: Agosto 2020.

SCHIMIGUEL, J.; FERNANDES, M. E.; OKANO, M. T. Investigando aulas remotas e ao vivo através de ferramentas colaborativas em período de quarentena e 
Covid-19: relato de experiência. Research, Society and Development, v. 9, n. 9, p. e654997387, 2020. DOI: http://dx.doi.org/10.33448/rsd-v9i9.7387.

Disponível

em: https://www.rsdjournal.org/index.php/rsd/article/vi ew/7387. Novembro 2020.

SENHORAS, E. M. Coronavírus e educação: análise dos impactos assimétricos. Boletim de Conjuntura, Boa Vista, v. 2, n. 5, p. 125-136, 2020. DOI: http://doi.org/10.5281/zenodo.3828085.

Disponível

em: https://revista.ufrr.br/boca/article/view/Covid-

19Educacao/0. Acesso em: Agosto 2020.

SILVA, D. S.; ANDRADE, L. A. P.; dos SANTOS, S.M.P. Teaching alternatives in pandemic times. Research, Society and Development, v. 9, $\mathrm{n}$. 9, p. e424997177, 2020.

DOI: http://dx.doi.org/10.33448/rsd-v9i9.7177.

Disponível

em: https://rsdjournal.org/index.php/rsd/article/view/71

77. Acesso em: Setembro 2020.

SPALDING, M. et. Al. Desafios e possibilidades para o ensino superior: uma experiência brasileira em tempos de COVID-19. Research, Society and Development, v. 9, n. $\quad$ 8, e534985970, 2020 DOI: https://doi.org/10.33448/rsd-v9i8.5970.

Disponível

em: https://www.rsdjournal.org/index.php/rsd/article/vi ew/5970. Acesso em: Outubro 2020.

TORRES, A. C. M.; Da COSTA, A. C. N.; ALVES, L. R. G. Educação e Saúde: reflexões sobre o contexto universitário em tempos de COVID-19. Scientific Electronic Library Online, São Paulo.
DOI: https://doi.org/10.1590/SciELOPreprints.640. Disponível em: https://preprints.scielo.org/index.php/scielo/preprint /view/640. Acesso em: Agosto 2020.

UNESCO - United Nations Educational, Scientific and Cultural Organization. "COVID-19 Educational Disruption and Response". UNESCO Website [06/05/2020]. Disponível em: https://en.unesco.org/covid19/educationresponse. Acesso em: Agosto 2020.

VERCELLI, L. C. A. Aulas remotas em tempos de Covid-19: a percepção de discentes de um programa de mestrado profissional em educação. Revista @ mbienteeducação, São Paulo, v. 13, n. 2, p. 47-60 Mai/Ago 2020 . DOI: https://doi.org/10.26843/ae19828632v13n22020p $47 \mathrm{a} 60$.

Disponível em: http://publicacoes.unicid.edu.br/index.php/ambient eeducacao/article/view/932. Acesso em: Outubro 2020.

VIEIRA, K. M.; POSTIGLIONI, G. F.; DONADUZZI, G.; PORTO, C. S.; KLEIN, L. L.. Vida de Estudante Durante a Pandemia: Isolamento Social, Ensino Remoto e Satisfação com a Vida. Revista Científica em Educação a Distância, v. 10, n. 3, p. e1147, 2020. DOI: 10.18264/eadf.v10i3.1147.

Disponível em: https://eademfoco.cecierj.edu.br/index.php/Revista/ article/view/1147. Acesso em Novembro 2020.

WORLD HEALTH ORGANIZATION (WHO). Novel Coronavirus (2019-nCoV). WHO, 21 de Jan. 2020. Disponível em: https://www.who.int/docs/defaultsource/coronaviruse/situation-reports/20200121-sitrep1-2019-ncov.pdf. Acesso em: Agosto 2020. 